\title{
Absorption, Diffraction and Free Space Path Losses Modeling for the Terahertz Band
}

\author{
Oluseun.D.Oyeleke ${ }^{\mathrm{a}}$, Dr Sadiq Thomas ${ }^{\mathrm{a}}$, Olabode Idowu-Bismark ${ }^{\mathrm{b}}$, Dr Petrus Nzerem, \\ Idris Muhammad ${ }^{\mathrm{c}}$ \\ ${ }^{a}$ Computer engineering department Nile University Nigeria \\ ${ }^{b}$ Covenant university \\ ${ }^{c}$ Petroleum and Gas Engineering Department ,Electrical and Computer engineering Nuhu Bamalli Poly \\ Zaria
}

Received: 24 September 2019; Accepted: 15 October 2019; Published: 08 February 2020

\begin{abstract}
With the explosive increase in the data traffic of wireless communication systems and the scarcity of spectrum, terahertz (THz) frequency band is predicted as a hopeful contender to shore up ultra- broadband for the forthcoming beyond fifth generation (5G) communication system. THz frequency band is a bridge between millimeter wave (mmWave) and optical frequency bands. The contribution of this paper is to carry out an in-depth study of the $\mathrm{THz}$ channel impairments using mathematical models to evaluate the requirements for designing indoor $\mathrm{THz}$ communication systems at 300GHz. Atmospheric absorption loss, diffraction loss and free space path loss were investigated and modeled. Finally, we discuss several potential application scenarios of $\mathrm{THz}$ and the essential technical challenges that will be encountered in the future $\mathrm{THz}$ communications. Finally, the article finds that propagating in the $\mathrm{THz}$ spectrum is strongly dependent on antenna gain.
\end{abstract}

Index Terms: Terahertz, Absorption loss, Diffraction loss, free path loss, Channel Model.

(C) 2020 Published by MECS Publisher. Selection and/or peer review under responsibility of the Research Association of Mode rn Education and Computer Science

* Corresponding author.

E-mail address: 


\section{Introduction}

Machine-type communications such as the Virtual reality applications, Internet of Things, Internet of Everything, vehicles Ado networks, Artificial intelligence, etc are expected to play an important role in the future wireless networks [1,2]. Especially in industry requiring automation, communication, safety for increased productivity. Edholm notes, is that we may someday see the end of wire line [3] and Moore's law is the observation that the number of transistors in a dense integrated circuit doubles about every two year [4]. This implies bandwidth scarcity in future due to the escalating rate of interconnected devices. Also, the current long term evolution advance (LTE/LTE-A) cellular networks cannot handle such gigantic connections since user terminals at the cell boundary experience very high interference, and therefore perform badly [5]. Furthermore, orthodox cellular systems are designed mainly for human users [1]. The use of a wide range of spectrum in the forth coming fifth generation (5G) communication network including the millimeter wave (MmWave) with its ability to provide large bandwidth from hundreds of $\mathrm{MHz}$ to several $\mathrm{GHz}$ range is able to offer much more bandwidth and connectivity than the traditional microwave communication below the 6GHz band [2]. Although the data rate provided by mmWave band can attain several Gbps, it is still not adequate to meet the requirement of the increasing data traffics in future wireless communications such as the future wireless local area networks (WLAN) and the wireless personal area networks (WPAN) systems with data rate of 10X Gbps [6]. Also, the least data rate of virtual reality (VR) devices is in the range of 10 Gbps while the data rate of uncompressed ultra-high-definition videos and 3D videos will reach 24 Gbps and 100 Gbps, respectively [6] leading to the need for an urgent research for much higher frequency resources.

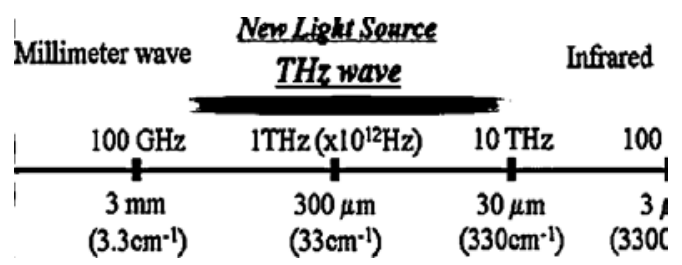

Fig.1. THz Spectrum

The THz spectrum as shown in Fig 1, also called sub-millimeter band covers the frequency range between $100 \mathrm{GHz}$ and $10 \mathrm{THz}$ and has a corresponding wavelengths between $3 \mathrm{~mm}$ and $30 \mu \mathrm{m}[7,8]$. This spectrum is the next frontier for high-bandwidth wireless communications because of its potential to provide up to Terabits per second (Tbps) link speed and to satisfy beyond fifth generation (5G) communication requirements such as high throughput and low latency. As such there is a strong interest from both academic and industrial sectors to develop efficient $\mathrm{THz}$ communication systems and related components [4]. THz band communication attracts such a great deal of attention due to its ability to achieve innovative applications for diverse scenarios, which has the ability to support the much higher speed data rates from tens of Gbps to several Tbps than mmWave band communication. Compared with wireless optical communication, the $\mathrm{THz}$ communication system is sensitive to the atmospheric effects in outdoor wireless communications. As for indoor wireless communications, it is much easier for $\mathrm{THz}$ frequency band to track the beam than optical frequency band, which will enormously affect the mobility of the wireless communication systems. Beyond that, there is another advantage that $\mathrm{THz}$ communication systems have the capability of utilizing the reflection paths to enhance the link gains in indoor applications [6]. As discussed above, a deeper research on $\mathrm{THz}$ communications is essential in the near future. $\mathrm{THz}$ communication indoor scenarios usually have many wireless blockers like furniture, hospital equipments, kitchen and other domestic apparatus. In other to design 
for practical deployment of such systems, it is necessary therefore to provide adequate analysis and channel models that properly describe the environment of deployment. In this paper, we model the absorption, diffraction and the free space path losses of the $\mathrm{THz}$ band in the indoor environment in the range. The rest of the paper is arranged as follows, Section II deals with the related works while we considered the $\mathrm{THz}$ propagation losses modeling in section III. Section IV considered the methodology while the result analysis is treated in section V. We conclude in section VI.

\section{RELATED WORKS}

The literature on terahertz band channel models is sparse. In [7], a deterministic channel model for line of sight (LOS) and non line of sight (NLOS) propagation in the 0.1 to $1 \mathrm{THz}$ frequency range was developed using ray tracing method and its channel capacity investigated. The authors of [9] [10] derived the impulse response for the $\mathrm{THz}$ band in the 0.1 to $10 \mathrm{THz}$ using the transmittance method. In the work of [11], a novel three dimensional time-varying $\mathrm{THz}$ channel model was developed that captures the idiosyncrasies in propagation parameters of indoor $\mathrm{THz}$ communications system. The authors of [12] proposed a channel model in the 0.5 to $1.5 \mathrm{THz}$ band to compute the path loss, propagation delay and noise for human tissues and body structures.

\section{MODELING TERAHERTZ PROPAGATION LOSSES}

\section{(A) Modeling Absorption Loss}

Various studies are being conducted to measure the absorption coefficient of $\mathrm{THz}$ waves using a mixture of technologies in photonics and electronics such as in $[13,14]$. In a few years' time $5 \mathrm{G}$ will be saturated in the mm wave and it will be imperative for THz wave's communication to be used. That is above $100 \mathrm{GHz}$. Atmospheric absorption as shown in Fig 2,however is a challenge that THz wireless transmission will have to contend with if we are to see the usefulness of it been deployed in practical usage [15]. Many atmospheric molecules like water and oxygen have been studies and known to absorb THz signals obviously due to their microscopic and sub-millimeter sizes of which $\mathrm{THz}$ tiny wavelengths cannot pass-over without being absorbed [15].

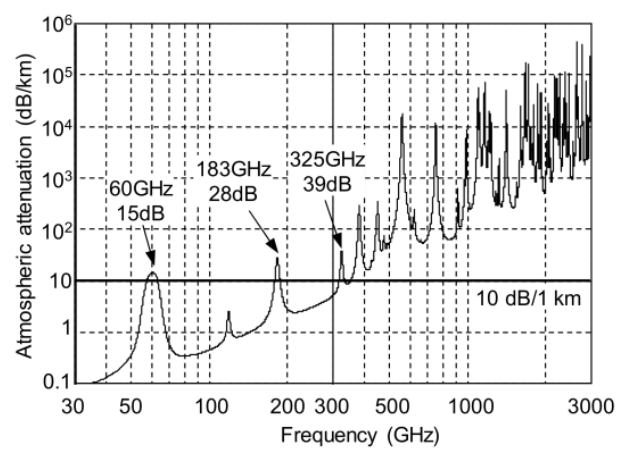

Fig .2. Attenuation in $\mathrm{dB} / \mathrm{km}$ of $\mathrm{THz}$ signal[22]

According to Beer-Lambert law [14], the transmittance of material sample is related to its optical depth and to its absorbance $\mathrm{A}$ as 


$$
T=\frac{\phi_{e}^{t}}{\phi_{e}^{i}}=e^{-r}
$$

Where $\phi_{e}^{t}$ is the radiant flux transmitted by that material sample and $\phi_{e}^{i}$ is the radiant flux received by that

material sample. Using the above to model our loss due to absorbtion in the Terahertz gap region we have transmittance as [14]

$$
T(f, d)=\frac{P_{r x}(f)}{P_{t x}(f)}=e^{-k_{a d}(f) d}
$$

Where $T(f, d)$ is transmittance, $P_{r x}(f)$ represents received power and $P_{t x}(f)$ represents transmit power and $K_{a c}$ is the absorption coefficient at frequency f, which describes the absorption coefficient and determines how far into a material light of a particular wavelength can penetrate before it is absorbed [9]. In a material with a low absorption coefficient, light is only poorly absorbed, and if the material is thin enough, it will appear transparent to that wavelength. The absorption coefficient depends on the material and also on the wavelength of light which is being absorbed. In the THz range, water has high absorption; the absorption coefficient of water is about $250 \mathrm{~cm}^{-1}$ at $1 \mathrm{THz}$.Water particles as vapor is the main source of molecular absorption in the frequency range above $200 \mathrm{GHz}$ [15]. The linearity of the Beer-Lambert law is restricted by chemical and instrumental factors. These includes deviations in absorptivity coefficients at high concentrations $(>0.01 \mathrm{M})$ due to electrostatic interactions between molecules in close proximity, the scattering of light due to particulates in the sample qs well as the fluorescence or phosphorescence of the sample. Therefore in the estimation of the absorption loss in the 275-400GHZ range, we utilize the proposed simplified channel model starts from the assumption of the Lorentz line shape holding for the relative loss. This line shape gives the easiest method to produce a molecular absorption model that only depends on volume mixing ratio of water (humidity) and frequency. The absorption coefficient can be estimated as

$$
k_{a c}=y_{1}\left(f, \mu_{w}\right)+y_{2}\left(f, \mu_{w}\right)+g(f)
$$

Combining (2) and (3), pathloss due to absorption is

$$
L_{a b s}\left(f, d, \mu_{w}\right)=e^{y_{1}\left(f, \mu_{w}\right)+y_{2}\left(f, \mu_{w}\right)+g(f)} .
$$

$\boldsymbol{\mu}_{w}$ is the water vapour volume mixing ratio which is given in terms of relative humidity $\phi$. The $\boldsymbol{\mu}_{w}$ has to be computed and is computed as [16]

$$
\boldsymbol{\mu}_{w}=\frac{\phi}{100} \frac{\boldsymbol{P}_{p w}\left(T_{s} p\right)}{P}
$$

where $P_{p w}$ is the partial pressure of water vapor at the condition of the saturated water vapor partial pressure $P_{p w}$ under pressure p and temperature T. The $P_{p w}$ can be computed by the buck equation. The vapor pressure of water is the pressure at which water vapor is in thermodynamic equilibrium with its condensed state. At higher pressures water would condense [16]. The water vapor pressure is the partial pressure of water vapor in any gas mixture in equilibrium with solid or liquid water. The water vapors volume mixing ratio is calculated with saturated water vapor partial pressure [17] as 


$$
P_{p w}=0.61221 e^{\left(\left(18.678-\frac{T}{234.84}\right)\left(\frac{T}{257.14+T}\right)\right)}
$$

Where $P_{p w}$ is the saturation vapor pressure in hPa, exp is the natural (i.e. base e) exponential function, $\mathrm{T}$ is the air temperature in degrees Celsius and the parameters in equation (4) are calculated based on [17] as;

$$
\begin{aligned}
& y_{1}\left(f, \mu_{w}\right)=\frac{y_{A}\left(\mu_{w}\right)}{y_{B}\left(\mu_{w}\right)+\left(\frac{f-c_{1}^{2}}{100_{c}}\right)^{2}} \\
& y_{2}\left(f, \mu_{w}\right)=\frac{y_{C}\left(\mu_{w}\right)}{y_{D}\left(\mu_{w}\right)+\left(\frac{f-c_{2}^{2}}{100_{c}}\right)^{2}}
\end{aligned}
$$

And

$$
g(f)=p_{1} f^{3}+p_{2} f^{2}+p_{3} f+p_{4}
$$

This model accuracy high for link distances of up to $1 \mathrm{~km}$ in standard atmospheric conditions.

\section{(B) Modeling Diffraction Loss}

Diffraction is a non-line-of-sight propagation phenomenon and is the "Bending” of wave fronts around obstacles. Diffraction occurs with all propagating waves, including sound waves, waves on water waves in materials and electromagnetic waves [18]. The effect of diffraction is generally only noticeable for waves where the wavelength is similar to the size of the diffracting object. Environments where propagation of waves travel might surely have obstacles which obstruct LOS signal between Tx and Rx. Idealized models for the evaluation of loss of signals due to diffraction by such obstacles are available. The form factor and geometric shape of the obstacles considered in these models maybe too idealized for real-life applications, but nevertheless, these models can serve as a good reference. One of such is the single knife-edge diffraction model. The model depicted in Figure 3 considers two idealized cases where a sharp obstacle is placed between the transmitter and the receiver Utilizing the geometric parameters in the figure, the diffraction loss can be evaluated with the help of a single, dimension-less quantity called Fresnel-Krichoff diffraction parameter - V. Based on the availability of information, any of equation (10) - (12) can be used to calculate this parameter as found in [19].

$$
\begin{gathered}
v=\sqrt[h]{\frac{2}{\lambda}\left(\frac{1}{d_{1}}+\frac{1}{d_{2}}\right)} \\
v=\theta \sqrt{\frac{2}{\lambda\left(\frac{1}{d_{1}}+\frac{1}{d_{2}}\right)}}
\end{gathered}
$$




$$
v=\sqrt{\frac{2 h \theta}{\lambda}=\sqrt{\frac{2 d}{\lambda}}} \propto_{1} \propto_{2}
$$

Where, $\mathrm{h}$ is the height of the top of the obstacle above the straight line joining the two ends of the path. If the height is below this line, $\mathrm{h}$ is negative. $\mathrm{d} 1$ and $\mathrm{d} 2$ are distances of the two ends of the path from the top of the obstacle $\theta$ is the angle of diffraction (radians) and its sign is the same as that of $h$. The angle $\theta$ is assumed to be less than about 0.2 radians, or roughly $12^{\circ}$ while $\alpha_{1}$ and $\propto_{2}$ are angles in radians between the top of the obstacle and one end as seen from the other end.

In indoor scenario when $\mathrm{THz}$ wireless is used for indoor wireless communication, there are usually domestic objects that can serve as obstacles and blockages for $\mathrm{THz}$ beam. It is not visible to assume LOS condition alone and hence imperative to consider diffraction losses to be able to model realistically $\mathrm{THz}$ communication characteristic. The model considers to study diffraction is the single sharp knife edge model which considers scenario where two sharp objects is in between $\mathrm{Tx}$ and $\mathrm{Rx}$, the diffraction loss can be calculated with the help of the Fresnel-kirchoff diffraction parameter v.

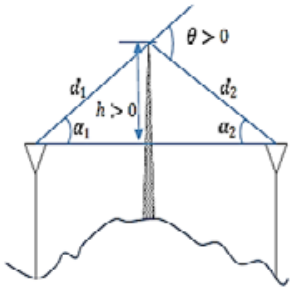

(a)

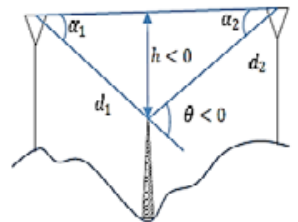

(b)

Fig.3. Diffraction single knife-edge obstacle having (a) postitive heights and (b) negative heights

Once the fresnel-kirchoff diffraction parameter has been estimated from the geometry, the signal level due to the single knife -edge can be evaluated by integrating the contributions provided by the unhindered parts of the wavefronts. The diffraction loss is hence obtained as [19]

$G(v)=20 \log _{10}\left[0.5 \sqrt{[1-C(v)-S(v)]^{2}+[C(v)-S(v)]^{2}}\right] d B$

Where $\mathrm{C}(\mathrm{v})$ and $\mathrm{S}(\mathrm{v})$ are the real and imaginary part of the complex Fresnel integral F(v) which is given as

$F(v)=\frac{(1+j)}{2} \int_{v}^{\infty} \exp \left[\frac{-j \pi t^{2}}{2}\right] d t$

The diffraction gain/loss can be computer by employing numerical methods but simplified for the case where $\mathrm{v}>-0.7$ the approximation in equation $\mathrm{xx}$ can be used

$\left.G(v)=6.9+20 \log _{10}\left(\sqrt{(v-0.1)^{2}+1}\right)+v-0.1\right) d B$

(C) Modeling FSPL 
Free-space path loss is often defined as the loss incurred by a radio signal as it travels in a straight line through a vacuum with no absorption or reflection of energy from nearby objects [15]. Free-space path loss assumes ideal atmospheric conditions so that no electromagnetic energy is actually lost or dissipated, it merely spreads out as it propagates away from the source, resulting in lower relative power densities. A more appropriate term for the phenomena is spreading loss. Free Space Path Loss (or FSPL) represents the amount of energy that a given radio wave loses as it travels through the air away from its source. Understanding FSPL will help us understand how far a Wi-Fi signal can go. It is also widely used by Wi-Fi survey tools to predict Wi-Fi signal propagation. FSPL is also applicable to any other waves using other frequencies. This loss is relative to 2 main components which are the frequency and distance. It is something that we can calculate by applying this mathematical formula ( $\mathrm{d}$ in $\mathrm{km}$ and $\mathrm{f}$ in $\mathrm{GHz}$ ):

$$
P_{r}(d)_{d B m}=P_{t}+G_{t}+G_{r}+20 \log _{10}(4 \pi d)-10 \log _{10}(L)
$$

\section{METHODOLOGY}

One major merit of the FSPL model is the probability of appending it with additional loss component so that other various propagation impairments can be included to create a more realistic scenario. These other losses gain coefficient can be multiplied by the estimated free space coefficient. Table 1 shows simulation parameters.

Table 1: Simulation parameters [20]

\begin{tabular}{c|c|c}
\hline S/N & Parameter & Value \\
\hline d1 & Distance of Tx to Obstacle & $40 \mathrm{~meter}$ \\
\hline $\mathrm{d} 2$ & Distance of Rx to Obstacle & $60 \mathrm{~meter}$ \\
\hline F & frequency & $300 \mathrm{GHz}$ \\
\hline Height of obstacle & 0.8 meters & \\
\hline T & Temperature in K & 302.5 \\
\hline P & Pressure & 101325.7 \\
\hline Relative humidity & $70 \%$ & $70 \%$ \\
\hline N & Path loss exponent & 2 \\
\hline
\end{tabular}

\begin{tabular}{l|ll}
\hline \multicolumn{1}{c|}{ Parameter } & \multicolumn{1}{c}{ Value } \\
\hline $\mathrm{C}_{1}$ & $10.835 \mathrm{~cm}^{-1}$ & \\
\hline $\mathrm{C}_{2}$ & $12.664 \mathrm{~cm}^{-1}$ & \\
\hline $\mathrm{p}_{1}$ & $5.54 \times 10^{-37} \mathrm{~Hz}^{-3}$ & \\
\hline $\mathrm{p}_{2}$ & $-3.94 \times 10-25 \mathrm{~Hz}^{-2}$ & \\
\hline $\mathrm{p}_{3}$ & $9.06 \times 10-14 \mathrm{~Hz}^{-1}$ & \\
\hline $\mathrm{p}_{4}$ & $-9.36 \times 10^{-3}$ & $(0.1303$ \\
\hline$y_{A}$ & $\begin{array}{l}0.2205 \quad \boldsymbol{\mu}_{w} \\
\left.\boldsymbol{\mu}_{\boldsymbol{w}}+0.0294\right)\end{array}$ \\
\hline$y_{B}$ & $\left(0.4093 \boldsymbol{\mu}_{\boldsymbol{w}}+0.0925\right)^{2}$ & \\
\hline$y_{C}$ & $2.014 \boldsymbol{\mu}_{\boldsymbol{w}}\left(0.1702 \boldsymbol{\mu}_{\boldsymbol{w}}+0.0303\right)$ \\
\hline$y_{D}$ & $\left(0.537 \boldsymbol{\mu}_{\boldsymbol{w}}+0.0956\right)^{2}$ & \\
\hline
\end{tabular}




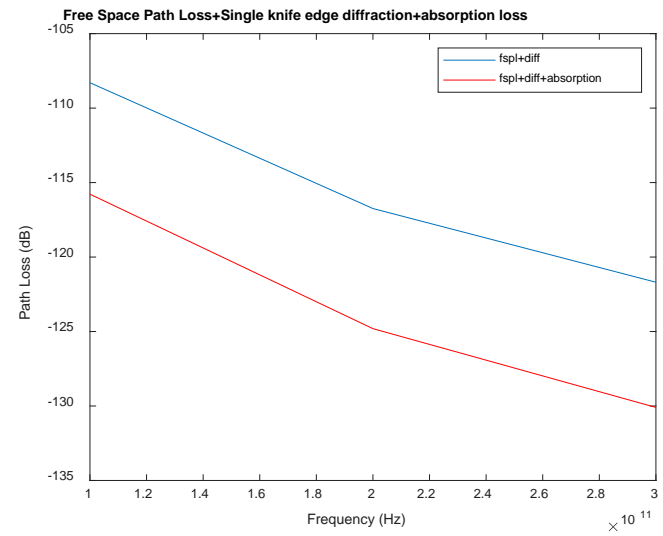

Fig.4. Pathloss as a function of the distance

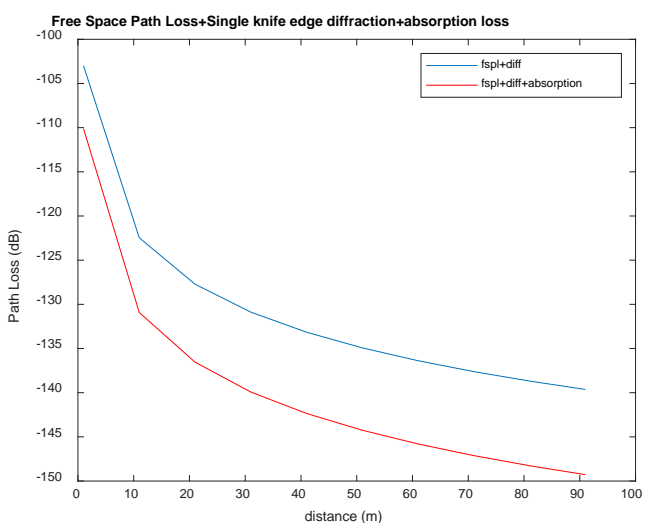

Fig.5. Pathloss as a function of the distance

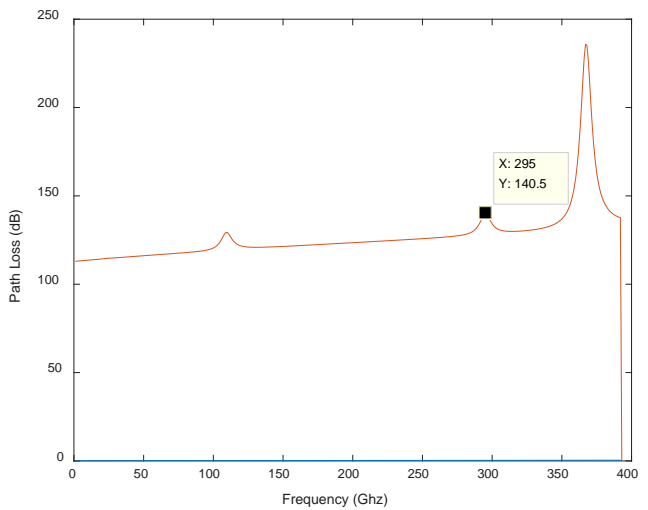

Fig.6. Pathloss simulation [21] 


\section{RESULT ANALYSIS}

The demonstration of the simulation behavior of the THz link in terms of Frequency, distance, Path gain and path loss with the combined effect of Absorption loss, diffraction and free space path loss is discussed in this section with various range of frequency from 100-300 GHz. For even though the model applied is proven to be valid between 275-400.The transmission distance, path lengths ranging from 1 to $100 \mathrm{~m}$ are considered, and graphs illustrates the path gain as a function of the frequency for different distances, assuming standard atmospheric conditions and $\mathrm{Gt}=\mathrm{Gr}=1$. The results derived with the presented path gain model are matching with the results derived by the Beer-Lambert law; thus, verifying the accuracy of the presented model. Validation was performed using the University of Buffalo $\mathrm{THz}$ nano online simulator as shown in Fig 6 and comparison done as shown in Table 2.

Table 2: Comprising with University of Buffalo THz simulator

\begin{tabular}{|l|l|}
\hline OUR MODEL & $\begin{array}{l}\text { UNIVERSITY OF } \\
\text { BUFFALO UB NANO }\end{array}$ \\
\hline Pathloss at 100Ghz is & Pathloss at 100Ghz is \\
116DB and 108dB & $130 \mathrm{~dB}$ \\
\hline Pathloss at $300 \mathrm{Ghz}$ is & Pathloss at 300Ghz is \\
$136 \mathrm{DB}$ and 121dB & $140 \mathrm{~dB}$ \\
\hline
\end{tabular}

Moreover, from Fig 4 and 5, it is apparent that for the transmission window under consideration, as the distance increases, the path gain decreases. We can see from the FSPL + Diffraction plot that from 30m we had $140 \mathrm{~dB}$ and $55 \mathrm{~m}$ it increased to $145 \mathrm{~dB}$ and hence just a $20 \mathrm{~m}$ difference introduced over $5 \mathrm{~dB}$ path loss, while within a $90 \mathrm{~m}$ difference we had a pathloss of over $50 \%$ increment. Steep pathloss value was observed between the first $10 \mathrm{~m}$.

When antenna gain was increased theoretically to $10 \mathrm{~dB}$ as shown in Fig 7, the table tabulated below shows the effect of antenna gain on pathloss improvement.Table3 shows the impact of increasing antenna gain to combact the severe path loss.

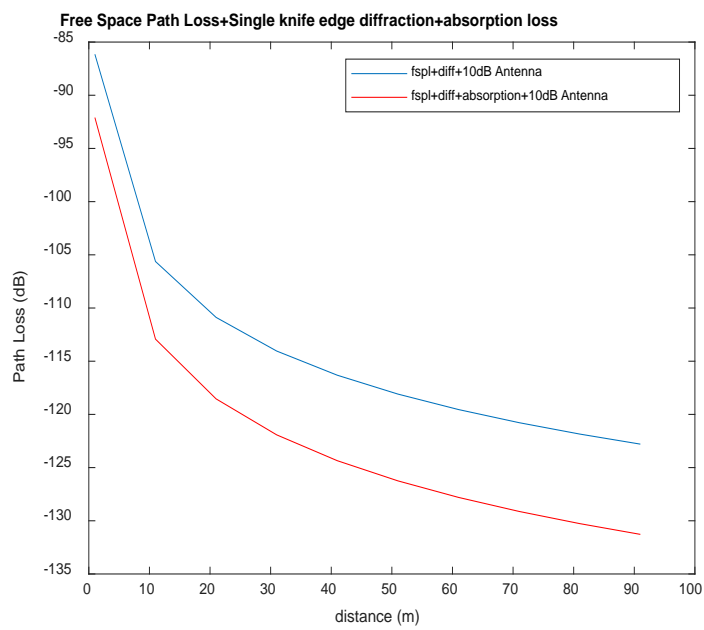

Fig.7. Pathloss simulation with improved antenna gain. 
Table 3: Impact of Increased Antenna Gain

\begin{tabular}{|l|l|}
\hline $\begin{array}{l}\text { Antenna Gain }=1 \mathrm{~dB} \text { at } 90 \mathrm{~m} \\
\text { (FSPL+DIF+ABSORPTION }\end{array}$ & $\begin{array}{l}\text { Antenna Gain } 10 \mathrm{~dB} \text { at } \\
90 \mathrm{~m}-\mathrm{FSPL}+\mathrm{DIF}+(\text { ABSORPTION) }\end{array}$ \\
\hline 150dB & $130 \mathrm{~dB}$ \\
\hline
\end{tabular}

\section{CONCLUSION}

In this research a channel system model was presented which included diffraction loss as well as free space path loss and absorption loss due to molecular interaction with $\mathrm{THz}$ waves. The model links used do not exceed $400 \mathrm{GHz}$ and were utilized to evaluate the performance of $\mathrm{THz}$ link in the presence of these path loss parameters. Validation was done using the university of buffalo model which we carried it through real life measurement and hence it is imperative that more measurement campaign must be performed to develop the more robust and realistic modeling (Mutaz et all) and recommended that the $\mathrm{THz}$ in various geographical location. Also the importance of developing low power and High gain directional antenna is inevitable if the usefulness of the $\mathrm{THz}$ band will be applied to build wireless communication devices. Terahertz sources are another area that must constantly bee researched, with the applicability of grapheme based sources and femto pulse lasers, the THz link is promising for future high speed applications.

\section{References}

[1] O. Idowu-Bismark, F. Idachaba, and A. A. A. Atayero, “A Survey on Traffic Evacuation Techniques in Internet of Things Network Environment,” Indian J. Sci. Technol., vol. 10, no. 33, pp. 1-11, 2017.

[2] O. Idowu-bismark, O. Kennedy, R. Husbands, and M. Adedokun, “5G Wireless Communication Network Architecture and Its Key Enabling Technologies,” vol. 12, no. April, pp. 70-82, 2019.

[3] E. S. Law, “Edholm’s law of bandwidth,” IEEE Spectr., vol. 41, no. 7, no. July, pp. 58-60, 2004.

[4] J. Wu, Y.-L. Shen, K. Reinhardt, H. Szu, and B. Dong, “A Nanotechnology Enhancement to Moore’s Law,” Appl. Comput. Intell. Soft Comput., vol. 2013, pp. 1-13, 2013.

[5] O. B. Idowu-Bismark, A. E. Ibhaze, and A. A. Atayero, "Mimo Optimization Techniques and Their Application in Maximizing Throughput for 3GPP HSPA+,” J. Wirel. Netw. Commun., vol. 7, no. 1, pp. 1-8, 2017.

[6] Z. Chen et al., “A Survey on Terahertz Communications,” China Commun., vol. 16, pp. 1-35, 2018.

[7] A. Moldovan, M. A. Ruder, I. F. Akyildiz, and W. H. Gerstacker, "LOS and NLOS Channel Modeling for Terahertz Wireless Communication with Scattered Rays,” pp. 388-392, 2014.

[8] Z. Miao, S. Member, Z. Hao, S. Member, and Y. Wang, “A 400-GHz High-Gain Quartz-Based Single Layered Folded Reflectarray Antenna for Terahertz Applications,” no. c, 2018.

[9] K. Tsujimura, K. Umebayashi, J. Kokkoniemi, and J. Lethomäki, “A Study on Channel Model for THz Band,” pp. 872-873, 2016.

[10] K. Tsujimura, K. Umebayashi, J. Kokkoniemi, J. Lehtomäki, and Y. Suzuki, “A Causal Channel Model for the Terahertz Band,” IEEE Trans. Terahertz Sci. Technol., vol. 8, no. 1, pp. 52-62, 2018.

[11] S. Nie and I. F. Akyildiz, "Three-Dimensional Dynamic Channel Modeling and Tracking for Terahertz Band Indoor Communications.” 
[12] K. Yang, Y. Hao, and A. Alomainy, “Channel Modelling of Human Tissues at Terahertz Band,” 2016 IEEE Wirel. Commun. Netw. Conf. Work., no. ComExCon, pp. 218-221, 2016.

[13] Q. T. Vien, M. O. Agyeman, T. A. Le, and T. Mak, "On the Nanocommunications at THz Band in Graphene-Enabled Wireless Network-on-Chip,” Math. Probl. Eng., vol. 2017, 2017.

[14] J. Kokkoniemi, J. Lehtomäki, and M. Juntti, “A discussion on molecular absorption noise in the terahertz band,” Nano Commun. Netw., vol. 8, pp. 35-45, 2015.

[15] M. . Paridah, A. Moradbak, A. . Mohamed, F. abdulwahab taiwo Owolabi, M. Asniza, and S. H. . Abdul Khalid, "We are IntechOpen, the world's leading publisher of Open Access books Built by scientists , for scientists TOP $1 \%$,” Intech, vol. i, no. tourism, p. 13, 2016.

[16] S. Shah and M. Ashraf, "Signal Path Loss Measurement for Future Terahertz Wireless Propagation Links,” vol. 5, no. 10, pp. 193-197, 2018.

[17] A. A. Boulogeorgos, E. N. Papasotiriou, J. Kokkoniemi, and J. Lehtom, "Performance Evaluation of THz Wireless Systems Operating in $275-400$ GHz Band."

[18] J. Kokkoniemi, P. Rintanen, J. Lehtomäki, and M. Juntti, "Diffraction effects in terahertz band Measurements and analysis,” 2016 IEEE Glob. Commun. Conf. GLOBECOM 2016 - Proc., 2016.

[19] P. Series, Propagation by diffraction P Series Radiowave propagation, vol. 11. 2009.

[20] Boulogeorgos, A.-A. A., Papasotiriou, E. N., Kokkoniemi, J., Lehtomaeki, J., Alexiou, A., \& Juntti, M. (2018). Performance Evaluation of THz Wireless Systems Operating in 275-400 GHz Band. 2018 IEEE 87th Vehicular Technology Conference (VTC Spring). doi: 10.1109/vtcspring.2018.8417891.

[21] Projects. (n.d.). Retrieved from https://unlab.tech/projects/

[22] Minoru Fujishima,Key Technologies for THz Wireless Link by Silicon CMOS Integrated Circuits, Photonics 2018, 5(4), 50; https://doi.org/10.3390/photonics5040050

\section{Authors’ Profiles}

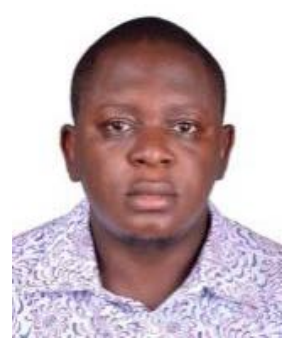

Oluseun D Oyeleke is a Master degree holder in Electronics and Communication Engineering and a current Ph.D. Student in Telecommunication. His focus is on 5G/6G wireless communication, Massive MIMO, MIMO and Machine learning for wireless telecommunication. He has over 12 years of lecturing experience and is currently an academic staff with the Nile University of Nigeria.

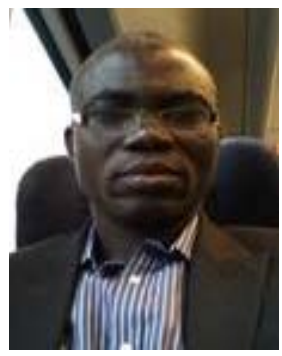

Olabode Idowu-Bismark is currently a PhD student in Covenant University, Ota, Ogun state Nigeria. He holds an M.Sc degree in Telecommunications Engineering from Birmingham University UK and a B.Eng degree in Electrical and Electronics Engineering from the University of Benin, Nigeria. Olabode is a ZTE University China certified RF engineer and a certified Optical Fibre/Transmission engineer, he has worked in various companies including Logic Sciences Limited and Primotek Systems Limited as an engineer, senior engineer and a technical director. A member of the IEEE, his research interest is in the area of wireless mobile communication, massive MIMO and the Internet of Things (IoT). 


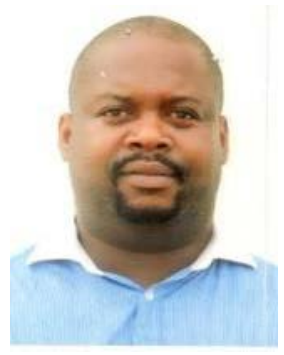

Sadiq Thomas was born in 1980, and is a Senior Lecturer, he has several yearse experience in academics from time spent completing his BEng in Computer Engineering and Numerical Modelling of EMATs his PhD Thesis. He has post-doctoral research and lecturing posts at various universities in the UK and Nigeria. HE completed his $\mathrm{PhD}$ in 2009 and his research was based on comprehensive finite element modelling of Electromagnetic Acoustic Transducer sensors used for Nondestructive testing of conductive materials particularly useful for pipeline maintenance.

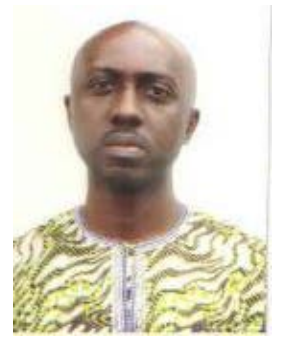

Dr Petrus Nzerem, DOB: 19 May 1980; Universities attended, Federal University of Technology Owerri 1998 - 2004 (BEng Polymer and Textile Engineering). University of Leeds, 2005 - 2006 (MSc Energy and Environment) University of Manchester 2008 2013 Chemical Engineering and Analytical Science.

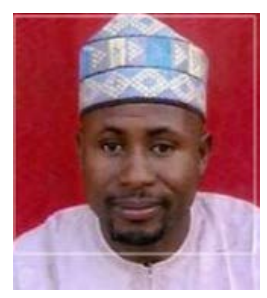

Engr Idris Muhammad is a graduate of Electrical engineering from Kaduna polytechnic; he proceeded for his PGD in Electrical at Bayero university Kano and also earned an Msc in same school in 2017.He consults for the state government on ICT and also lectures at the Nuhu bammalli Polytechnic Zaria.

How to cite this paper: Oluseun.D.Oyeleke, Sadiq Thomas, Olabode Idowu-Bismark, Petrus Nzerem, Idris Muhammad. "Absorption, Diffraction and Free Space Path Losses Modeling for the Terahertz Band", International Journal of Engineering and Manufacturing(IJEM), Vol.10, No.1, pp.54-65, 2020. DOI: 10.5815/ijem.2020.01.05 